\title{
THE EFFECTS OF IONIZING RADIATION ON POWER-MOSFET TERMINATION STRUCTURES*
}

\author{
K.R. Davis $\uparrow$, R.D. Schrimpf, F.E. Cellier, and K.F. Galloway \\ Department of Electrical and Computer Engineering \\ University of Arizona \\ Tucson, AZ 85721
}

\author{
D.I. Burton and C.F. Wheatley, Jr. \\ Harris Semiconductor \\ Mountaintop, PA 18707
}

\section{ABSTRACT}

The effects of ionizing radiation on power-MOSFET termination structures were examined through two-dimensional simulation. A wide range of sensitivity to surface-charge density was found for various devices employing floating field rings and/or equipotential field plates. Termination structures that were both insensitive to surface charge and possessed a high breakdown voltage were identified. The results were compared with measurements made on selected structures.

\section{INTRODUCTION}

The maximum breakdown voltage obtainable for a $p-n$ junction of specified doping concentration occurs for a planar structure [1]. In actual devices utilizing planar diffusion technology, however, the high-voltage junction must intersect the surface at some finite position. The resulting junction curvature compresses the equipotential lines where the junction bends to the surface and increases the peak electric field. Junction-termination structures are used in power devices to reduce the peak electric field and to allow the breakdown voltage to approach its ideal one-dimensional value. The most commonly used termination methods are field rings and field plates.

Avalanche multiplication is the physical mechanism that causes the primary breakdown of high-voltage reverse-biased $p-n$ junctions. Breakdown occurs when the electric field in the junction depletion region increases to the point at which the impact ionization rate approaches infinity. The most important factor that influences the magnitude and location of the peak electric field in planar semiconductor technology is the method used to terminate the junction [1-3]. The objective of the different termination methods is to reduce the peak electric field either at the surface or at the curved junction regions in the bulk.

The drain-source breakdown voltage of power MOSFETs

*

Work supported in part by the Defense Nuclear Agency under contract DNA-001-88-C-0004 and by the Naval Weapons Support Center.

$\dagger$ Now at Sandia National Laboratories, Albuquerque, New Mexico. is strongly affected by ionizing radiation $[4,5]$. This is a result of the introduction of trapped oxide and interface charge in the field oxide. These trapped charges alter the potential at the surface of the junction and, in turn, become a part of the junction termination. Therefore, radiation-induced charge affects the breakdown voltage of high-voltage junctions.

This paper reports on work designed to examine the effects of ionizing radiation on the most commonly used power-MOSFET termination structures. Simulations were conducted using computer code specifically designed for this purpose. The results are presented and verified with measurements made on selected structures. Insights into the design of optimum termination structures are obtained.

\section{HIGH-WOLTAGE TERMINATION TECHNIQUES}

Junction curvature significantly reduces the breakdown voltage of $p$ - $n$ junctions because of the increase of the electric field in the curved area [1]. This curvature is dependent on the junction depth, the ratio of the radius of curvature to depletion width, and the relative doping concentration. High voltage devices rely on the minimization of this curvature through the use of appropriate junction termination structures.

\section{A Floating Field Rings}

Figure 1 shows the cross-section of a power-MOSFET which includes one floating field ring. Field rings are often used to reduce the electric-field magnitude in the vicinity of the curved regions of the junction. The structure is designed

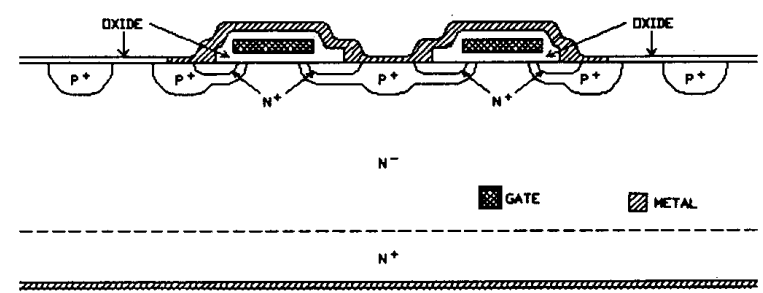

Fig. 1 Cross-section of power MOSFET with one floating field ring. 
to allow the reverse-biased junction's depletion region to punch-through to the ring well before the critical electric field for breakdown is attained. The effectiveness of the floating field ring is the greatest for high-voltage devices fabricated with shallow junctions [3]. When no surface charge is present, punch-through occurs at the surface. With the addition of surface charge, the depletion region may be compressed at the surface. In this case, for the field ring to be effective, punch-through must take place in the bulk silicon before the surface electric field becomes so high that breakdown occurs.

Optimally placing the field ring can result in a doubling of the breakdown voltage [7]. This optimal spacing depends on the oxide thickness and doping concentration. Adding surface charge in $\mathrm{N}$-channel power-MOSFETs decreases the breakdown voltage $[4,5]$. When the surface charge is fixed, it is possible to determine the optimal location of the field ring. When the surface charge is varied, however, the optimal location of the field ring changes, also.

\section{B. Equipotential Field Plates}

A field plate is a conductor placed above, and insulated from, the junction. It is usually connected to one side of the junction and is an extension of the junction metallization over the oxide as shown in Fig. 2. A field plate alters the surface potential by acting through the oxide layer to force the depletion layer at the surface to extend beyond the edge of the field plate. This "stretching out" of the depletion region reduces the depletion layer curvature and, in turn, reduces the peak electric field. It is common to use a field plate in conjunction with floating field rings by placing the plate on the main junction with floating rings beyond the junction or using a field plate on the last floating ring as shown in Fig. 3.

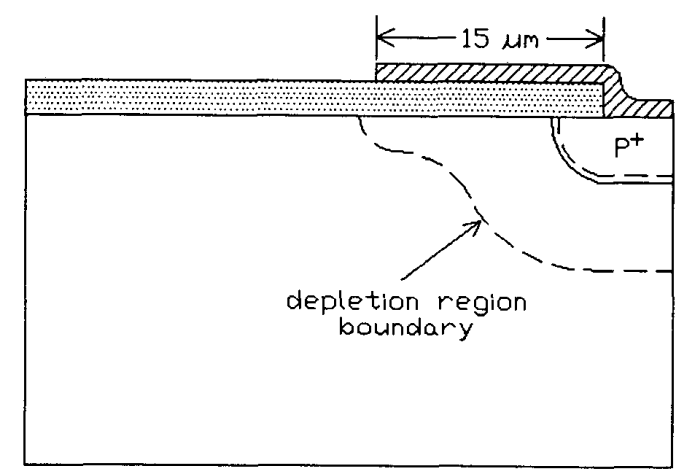

Fig. 2 Field-plate termination structure (After Baliga [3]).

\section{METHOD OF SIMULATION}

Computer code designed to analyze breakdown phenomena in high-voltage devices [6] has been modified to

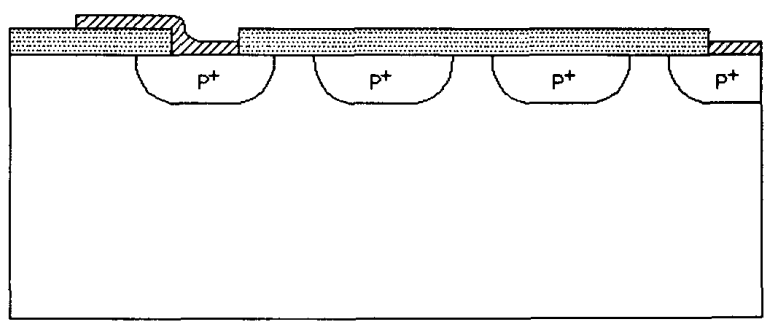

Fig. 3 Termination structure using combination of field rings and field plates. (After Baliga [3]).

simulate the effects of ionizing radiation on the breakdown voltage of power-MOSFETs. The code, entitled ASEPS (Arizona SEmiconductor Power device Simulator), solves Poisson's equation for the electrostatic potential distribution in two dimensions for an arbitrary device, subject to appropriate boundary conditions. Unlike many general-purpose simulators, ASEPS neglects the contribution of mobile charge to Poisson's equation. This approximation is very accurate for strongly reverse-biased junctions. This simplification results in reduced simulation time and improved convergence of the solution, without significant sacrifice in accuracy. Average simulation times on a Digital Equipment Corporation MicroVAX 3600 range from 5 minutes for simple structures to 25 minutes for more complex structures. ASEPS interfaces with AutoCAD [13] to produce detailed drawings of the potential distribution and electric field distribution suitable for analysis.

The radiation-induced areal surface-charge density, $N_{s s}$, used in the difference equations is an input parameter to the ASEPS code. In this work, $\boldsymbol{N}_{s s}$ represents the algebraic difference between the positive oxide trapped charge $\left(N_{o t}\right)$ and the negative interface trapped charge $\left(N_{i t}\right)$ and is taken to be positive. ASEPS draws a rectangular box around each node of the discretized domain of the structure being simulated, and this surface-charge density is only included in the difference equations for those nodes at the surface. A 200 $\times 200$ grid is practical for describing the simulation domain of the structure. The Newton successive overrelaxation method of solving the resulting nonlinear equations is employed until convergence of a solution is reached.

ASEPS uses the ionization integral,

$$
1-\frac{1}{M_{p}}=\int_{0}^{x} \alpha_{p} \exp \left[\int_{0}^{x^{\prime}}\left(\alpha_{n}-\alpha_{p}\right) d x^{\prime \prime}\right] d x^{\prime}
$$

to calculate the multiplication factor for determination of avalanche breakdown [7]. In Eq. 1, $M_{p}$ is the multiplication factor for holes, $\alpha_{p}$ is the ionization rate for holes, $\alpha_{n}$ is the ionization rate for electrons, and $x$ is the depletion-layer 
width of the ionization path. The ionization path is determined by starting from the peak electric field location and following the potential gradient line in both directions [8]. Ionization rates are from Sze and Gibbons [9]. Breakdown was assumed to occur when the multiplication factor for holes, $M_{p}$, reached the value of $10[10]$.

\section{RESULTS}

For a one-dimensional abrupt junction, breakdown voltage is approximated by the following equation [7],

$$
V_{B D}=\frac{\varepsilon E_{\max }^{2}}{2 q N_{B C}},
$$

where $\varepsilon$ is the permittivity, $E_{\max }$ is the maximum electric field, $q$ is the elementary charge, and $N_{B C}$ is the background (substrate) doping concentration. A one-dimensional structure was simulated using ASEPS. The doping profile used was approximately Gaussian with a surface concentration of $1 \times 10^{18} \mathrm{~cm}^{-3}$. The substrate concentration was approximately $0.9 \times 10^{15} \mathrm{~cm}^{-3}$. This doping profile was obtained from spreading resistance measurements made on an existent device. ASEPS calculated the breakdown voltage to be $327 \mathrm{~V}$ with an $E_{\max }$ of $3.0 \times 10^{5} \mathrm{~V} / \mathrm{cm}$, while Eq. (2) predicts that the breakdown voltage will be $324 \mathrm{~V}$. Thus for this simple example, there is less than a $1 \%$ difference between Eq. (2) and simulation.

A simple $p$ - $n$ junction formed by diffusion is considered next. Fig. 4a shows a cross section of this structure with equipotential lines calculated by ASEPS superimposed. The junction depth is $5 \mu \mathrm{m}$, the oxide thickness is $0.7 \mu \mathrm{m}, N_{s s}$ is zero, and the doping profile described above is used. The solid circle in Fig. 4a shows the location of the peak electric field. The value of the field is $3.5 \times 10^{5} \mathrm{~V} / \mathrm{cm}$. Calculation of the multiplication factor along the ionization path indicated that breakdown occurred for a reverse-bias voltage of $194 \mathrm{~V}$. $194 \mathrm{~V}$ is only $59 \%$ of the optimal $327 \mathrm{~V}$ that might be achieved in an ideal junction with $N_{B C}=0.9 \times 10^{15} \mathrm{~cm}^{-3}$.

Fig. 4b shows the same cross section except that the device was simulated with $N_{s s}=1 \times 10^{12} \mathrm{charges} / \mathrm{cm}^{2}$. Because the net charge in the oxide has been taken to be positive, the carrier density in the $n^{-}$region near the surface increases. This causes a decrease in depletion layer width and the crowding of the potential lines at the surface. The solid circle, again, shows the peak electric field location, which has moved to the surface. This location of the peak electric field results in a drastic reduction in breakdown voltage. A plot of breakdown voltage versus surface-charge density for this device can be found as the curve labeled "0 Rings" in Fig. 8.

The first technique considered to improve the breakdown voltage is the addition of one floating field ring optimally spaced from the main junction. This device, with the potential

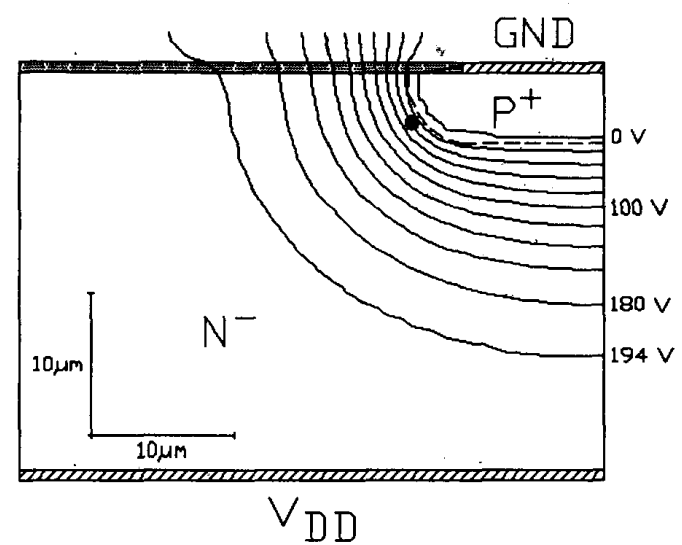

(a)

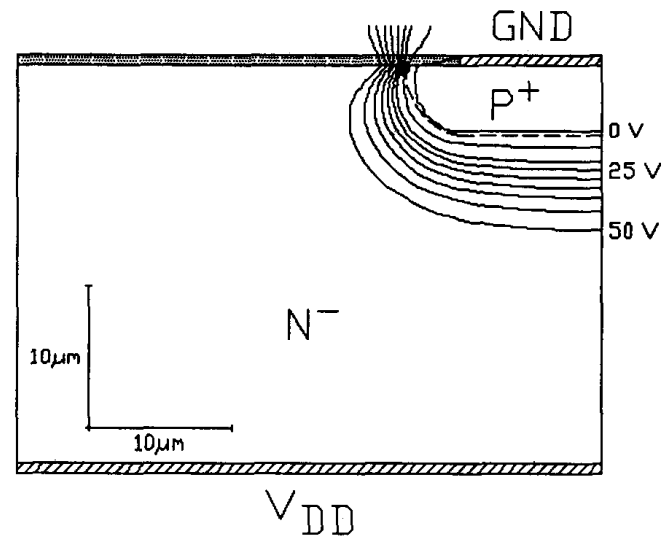

(b)

Fig. 4 Potential distribution for simple two-dimensional $p-n$ junction. (a) $N_{s s}=0, V_{B D}=194 \mathrm{~V}$. (b) $N_{s s}=$ $10^{12} \mathrm{~cm}^{-2}, V_{B D} \stackrel{s s}{=} 92 \mathrm{~V}$.

distribution for $N_{s s}=0$, is shown in Fig. 5 where optimal spacing is achieved because the high electric field spots (circles) appear on both junction curvatures simultaneously indicating that both junctions broke down. From the computer simulations, it was verified that this spacing resulted in the highest breakdown voltage. This optimal spacing was $9.5 \mu \mathrm{m}$

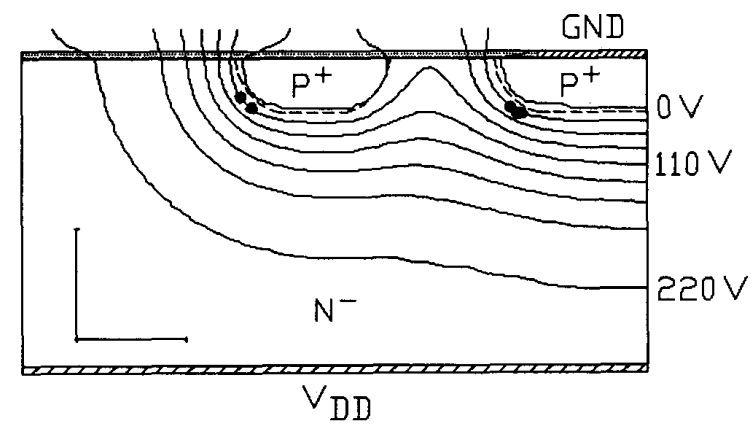

Fig. 5 Potential distribution for one-ring termination structure $\left(N_{s s}=0, V_{B D}=250 \mathrm{~V}\right)$. 
between rings after lateral diffusion. Figure 8 , where breakdown voltage of this one-ring device is plotted versus surface charge, indicates that for $N_{s s}=9 \times 10^{11} \mathrm{~cm}^{-2}$ or greater, the breakdown curve followed that of the zero-ring device. This happened because punch-through to the field ring did not take place before breakdown occurred at this value of $N_{s s}$. Better results were obtained for a three-ring device. Again, the sharp bends in the curve plotted in Fig. 8 are a result of the punch-through phenomenon not reaching a ring before breakdown occurred. The spacing of the rings was kept fixed in determining the optimal spacing in this three-ring device. It was found that this distance was $7.5 \mu \mathrm{m}$ and this device structure is shown in Fig. 6, with the overlaid potential distribution simulated for $N_{s s}=0$.

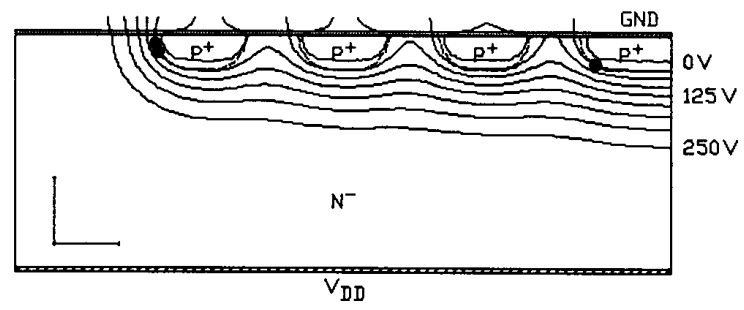

Fig. 6 Potential distribution for three-ring termination structure $\left(N_{s s}=0, V_{B D}=278 \mathrm{~V}\right)$.

Variable ring spacing increases the breakdown voltage of devices having more than one ring. For avalanche breakdown to occur on all rings simultaneously, the spacing between different pairs of rings must be non-constant. The seven-ring breakdown curve in Fig. 8 is for a device with this type of spacing. Fig. 7 shows this device, with the potential distribution for $N_{s s}=0$, where the spacing between the first ring and the main junction is $2.0 \mu \mathrm{m}$, increasing to $12.0 \mu \mathrm{m}$ between the last pair of rings. Experimental measurements on this device indicated no degradation of the $268 \mathrm{~V}$ breakdown voltage for total-dose levels up to $1 \mathrm{MRad}(\mathrm{Si})$. ASEPS calcu lated the breakdown voltage to be $281 \mathrm{~V}$ which is in error by only $5 \%$. From the potential distribution in Fig. 7 , it can be seen that the depletion layer depth below each ring gradually decreases as one moves farther from the main junction. The potential difference between a ring and the bulk material decreases as the distance of the ring from the main junction increases. This gradual decrease in depletion layer depth causes the termination to approximate the performance of a parallel-plane junction.

The measured breakdown voltage of a six-ring structure is plotted versus surface-charge density in Fig. 9. Each data point represents the average of six samples. The total dose has been converted to surface-charge density using the formula [12],

$$
N_{s s}=D t_{a x} G F
$$

where $D$ is the total dose, $t_{o x}$ is the oxide thickness, $G$ is the generation rate of electron-hole pairs per unit volume, and $F$ is the fraction of holes transported and trapped at the interface. An appropriate value of $F$ was used in this simple model ( $F=0.001)$ to calibrate the experimental data to the simulation. A more sophisticated model or additional experiments could be used to establish more precisely the relation between total dose and surface-charge density if required. In most situations, however, it is sufficient to minimize the dependence of breakdown voltage on surface-charge density.

The field plate/field ring combination shown in Fig. 10a had an initial breakdown voltage that was higher than that of a one-ring structure, but the advantage disappeared with increased surface charge. This is due to the fact that punchthrough does not occur for higher charge densities and the plate has no effect (Fig. 10b). These results are presented in Fig. 11 where a comparison of this plate structure is made with the one-ring structure that does not use a field plate.

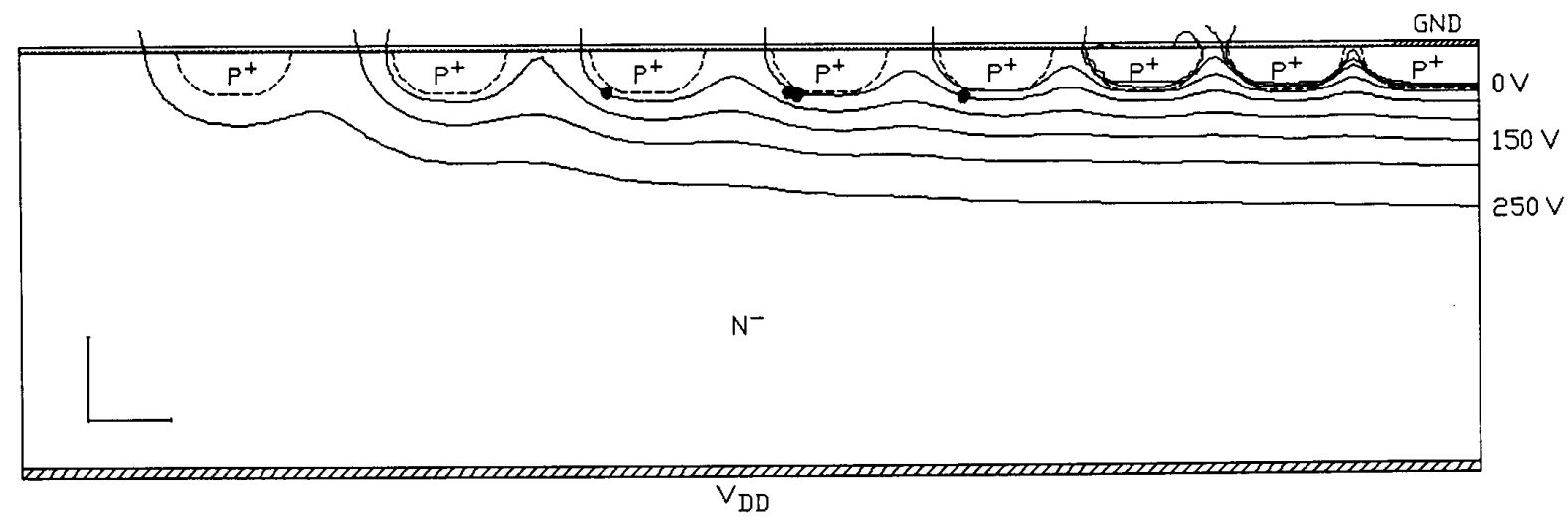

Fig. 7 Potential distribution for seven-ring termination structure $\left(N_{\mathrm{ss}}=0, \mathrm{~V}_{\mathrm{BD}}=281 \mathrm{~V}\right)$. 


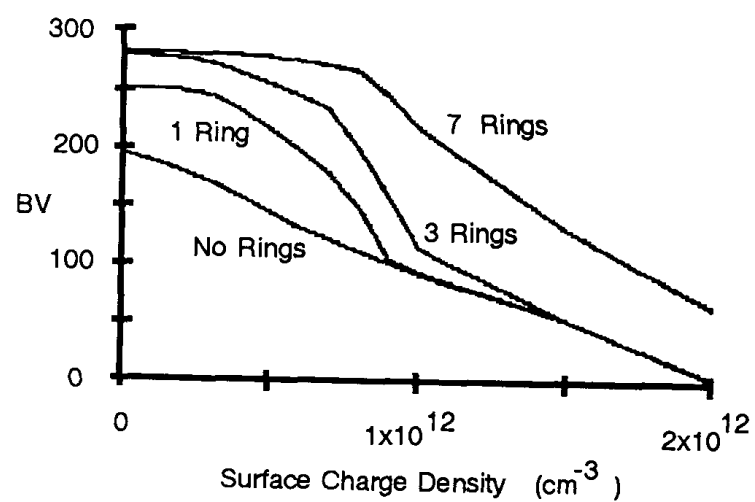

Fig. 8 Breakdown voltage vs. surface-charge density for various numbers of floating rings.

\section{CONCLUSIONS}

The breakdown behavior of typical power-MOSFET termination structures was examined as a function of radiation-induced surface charge, and the results for selected structures were verified experimentally. The specific results presented are a function of doping profile and oxide thickness, but the approach is readily applicable to structures with other values of these parameters. A wide range of sensitivity to radiation-induced charge was found for different termination structures.

For a field plate to be effective in increasing the breakdown voltage, it is necessary to use an oxide thickness sufficiently large so that the depletion layer curvature is reduced and the peak electric field is moved lower in the semiconductor bulk. With a large oxide thickness, though, the structure is very sensitive to surface charge. This causes field-plate terminations to degrade severely in radiation environments because field plates modify the potential distribution in the semiconductor by acting through an intervening dielectric layer. Any charge that is added in this dielectric layer produces a significant change in the potential distribution in the semiconductor.

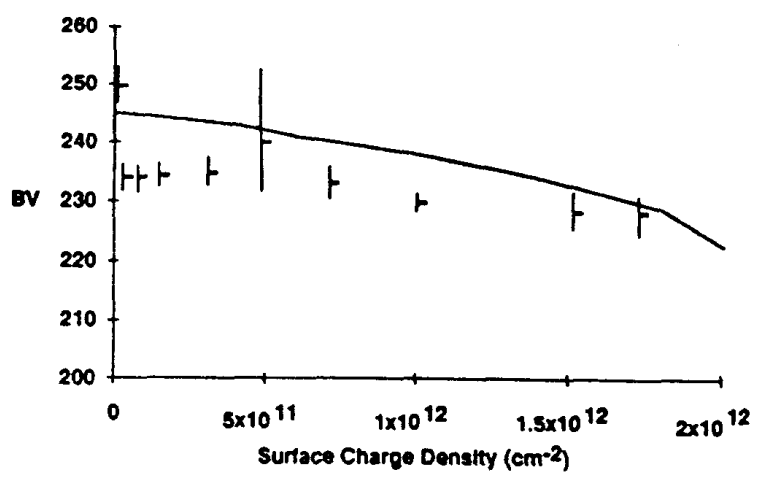

Fig. 9 Breakdown voltage vs. surface-charge density for a sixring structure. Each data point represents the average of six samples. The line represents simulation results.
To achieve a high breakdown voltage and low sensitivity to surface charge, a device employing multiple field rings was found to work well. With just one ring, the breakdown voltage was substantially increased and sensitivity to surface charge was reduced. The optimal spacing of this ring was found to be $9.5 \mu \mathrm{m}$ such that the breakdown voltage was maximized and the peak electric field minimized for the given impurity profile. The optimal spacing of a three-ring device was $7.5 \mu \mathrm{m}$ between rings spaced uniformly. For avalanche multiplication to occur on all rings simultaneously, the spacing of the rings should gradually increase as distance from the main junction increases. This method of spacing the rings was used in the seven-ring device where the first ring was spaced $2.0 \mu \mathrm{m}$ from the main junction.
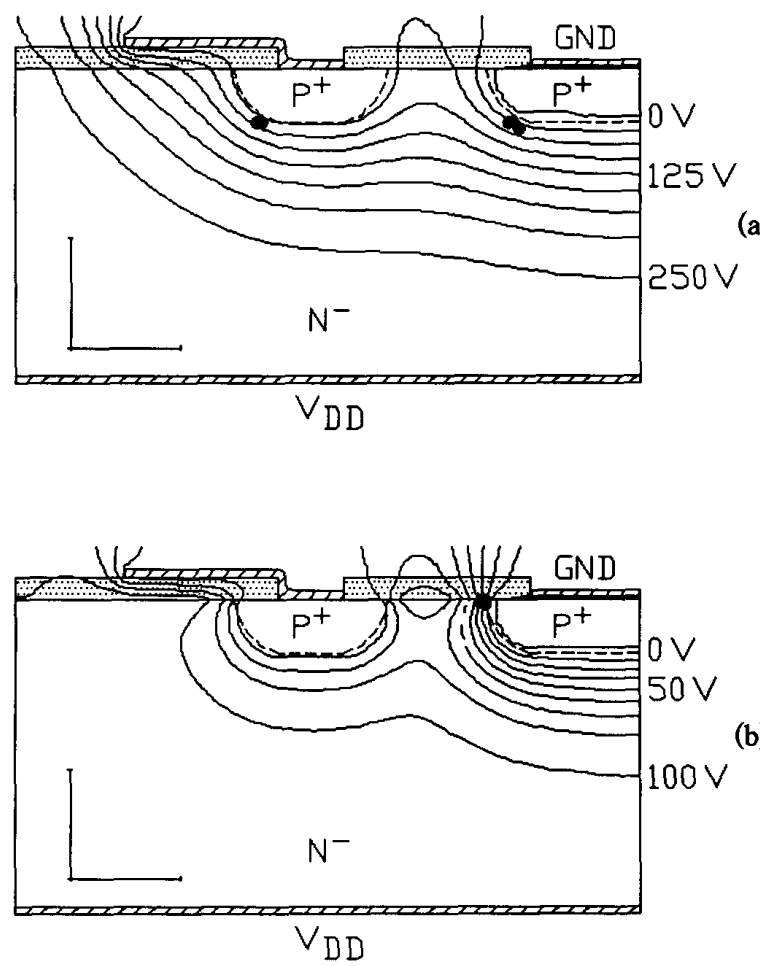

Fig. 10 Potential distribution for combination ring/plate structure. (a) $N_{s s}=0, V_{B D}=270 \mathrm{~V}$. (b) $N_{s s}=$ $10^{12} \mathrm{~cm}^{-3}, V_{B D}=92 \mathrm{~V}$.

The trend is that as the number of rings in the device is increased, the spacing between these rings should steadily decrease. The best results are obtained for devices with a large number of rings and very little spacing between them.

There is, however, a tradeoff between die area and the desired increase in breakdown voltage and the desired insensitivity to surface charge. The increase in breakdown voltage of an eight-ring device over a seven-ring device is very slight. The most appropriate number of rings will be de- 


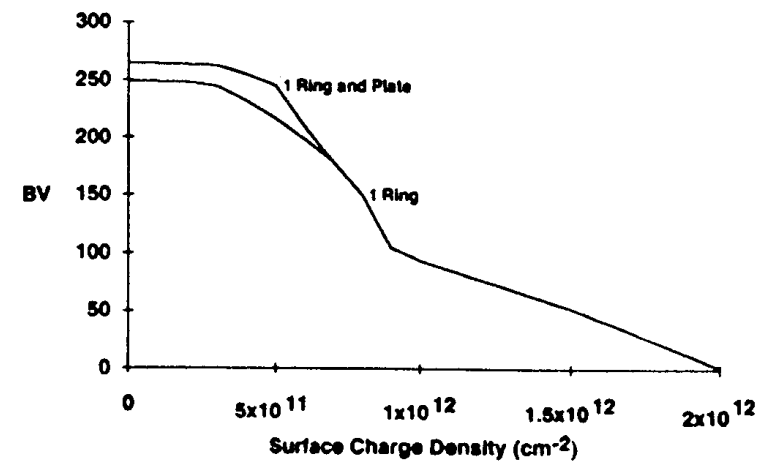

Fig. 11 Breakdown voltage vs. surface charge density for a one-ring structure and a one-ring structure with a field plate.

termined by the application and by the radiation environment in which the device is to be deployed.

Great care must be taken when designing a power MOSFET for use in an ionizing radiation environment. ASEPS has proven to be a valuable tool for this type of design work [14]. Of the structures examined, multiple floating rings had the least sensitivity to radiation-induced charge. The spacing between these rings is critical because radiationinduced charge can render the outer rings ineffective if the distance between them is too large. By using ASEPS with some initial insight as to what the spacing should be, one has a good starting point for determining optimal ring spacing.

\section{ACKNOWLDGEMENTS}

We would like to express thanks to Burr-Brown Corporation for the use of computer resources and an early involvement in the development of the ASEPS code. Also, we would like to thank Chi-min Yen and Leonghin Tan for their contributions to the development of the original code. Finally, we appreciate the interest of Tom Ellis, Jeff Titus, Dale Platteter, and Lew Cohn in this problem.

\section{REFERENCES}

1. B.J. Baliga, "Silicon Power Field Controlled Devices," in: Silicon Integrated Circuits, Part B, (D. Kahng, ed.), Academic Press, New York, pp. 158-174, 1981.

2. B.J. Baliga, "High-Voltage Device Termination Techniques - A Comparative Review," IEE Proc., vol. 129, pp. 173-179, 1982.
3. B.J. Baliga, Modern Power Devices. John Wiley, New York, 1987.

4. D.L. Blackburn, J.M. Benedetto, and K.F. Galloway, "The Effects of Ionizing Radiation on the Breakdown Voltage of Power MOSFETs," IEEE Trans. Nuclear Science, vol. NS-30, pp. 4116-4121, 1983.

5. R.D. Pugh, A.H. Johnson, and K.F. Galloway, "Characteristics of the Breakdown Voltage of Power MOSFETs After Total Dose Irradiation," IEEE Trans. Nuclear Science, vol. NS-33, pp. 1460-1464, 1986.

6. Q.-M. Wu, C.-M. Yen, and F.E. Cellier, "Analysis of Breakdown Phenomena in High-Voltage Devices," Trans. of SCS, to appear.

7. R.M. Warner, Jr. and B.L. Grung, Transistors: Fundamentals for the Integrated Circuit Engineer. John Wiley, New York, 1983.

8. M.S. Adler, V.A.K. Temple, A.P. Ferro, and R.C. Rustay, "Theory and Breakdown Voltage for Planar Devices with a Single Field Limiting Ring," IEEE Trans. Electron Devices, vol. ED-24, pp. 107-113, 1977.

9. S.M. Sze and G. Gibbons, "Avalanche Breakdown Voltages of Abrupt and Linearly Graded $p$-n Junctions in Ge, Si, GaAs, and GaP," Appl. Phys. Lett., vol. 8, pp. 111-135, 1966.

10. K. Hwang and D.H. Navon, "Breakdown Voltage Optimization of Silicon $p-\pi-v$ Planar Junction Diodes," IEEE Trans. Electron Devices, vol. ED-31, pp. 1126$1135,1984$.

11. V. Boison, M. Le Helley, and J.-P. Chante, "Computer Study of a High-Voltage $p-\pi-n-n^{+}$Diode and Comparison with a Field-Limiting Ring Structure," IEEE Trans. Electron Devices, vol. ED-33, pp. 80-84, 1986.

12. J.M. McGarrity, "Considerations for Hardening MOS Devices and Circuits for Low Radiation Doses," IEEE Trans. Nuclear Science, vol. NS-27, pp. 1739-1744, 1980.

13. Autodesk, Inc., The AutoCAD Reference Manual. Sausalito, California, 1987.

14. K.R. Davis, "Two-Dimensional Simulation of the Effects of Total Dose Ionizing Radiation on Power-MOSFET Breakdown," University of Arizona, Tucson, M.S.E.E. Thesis, 1989. 